\title{
BVES is a novel interactor of ANO5 and regulates myoblast differentiation
}

\author{
Haiwen Li ${ }^{1}$, Li Xu' ${ }^{1}$, Yandi Gao ${ }^{1}$, Yuanbojiao Zuo ${ }^{1,2}$, Zuocheng Yang ${ }^{2}$, Lingling Zhao ${ }^{2}$, Zhiheng Chen², \\ Shuliang Guo ${ }^{1}$ and Renzhi Han ${ }^{1 *}$ (1)
}

\begin{abstract}
Background: Anoctamin 5 (ANO5) is a membrane protein belonging to the TMEM16/Anoctamin family and its deficiency leads to the development of limb girdle muscular dystrophy R12 (LGMDR12). However, little has been known about the interactome of ANO5 and its cellular functions.

Results: In this study, we exploited a proximal labeling approach to identify the interacting proteins of ANO5 in C2C12 myoblasts stably expressing ANO5 tagged with BiolD2. Mass spectrometry identified 41 unique proteins including BVES and POPDC3 specifically from ANO5-BiolD2 samples, but not from BioID2 fused with ANO6 or MG53. The interaction between ANO5 and BVES was further confirmed by co-immunoprecipitation (Co-IP), and the N-terminus of ANO5 mediated the interaction with the C-terminus of BVES. ANO5 and BVES were co-localized in muscle cells and enriched at the endoplasmic reticulum (ER) membrane. Genome editing-mediated ANO5 or BVES disruption significantly suppressed C2C12 myoblast differentiation with little impact on proliferation.
\end{abstract}

Conclusions: Taken together, these data suggest that BVES is a novel interacting protein of ANO5, involved in regulation of muscle differentiation.

Keywords: ANO5, BioID2, BVES, Muscle differentiation, Muscular dystrophy, Proximity labeling

\section{Introduction}

ANO5 is a member of the TMEM16/Anoctamin family with putative chloride channel function and/or phospholipid scrambling activity [1-3]. Genetic mutations in the ANO5 gene cause recessive LGMDR12 and Miyoshi myopathy type 3 (MMD3) characterized by progressive muscle weakness and degeneration, and dominant gnathodiaphyseal dysplasia (GDD) [4]. Ano5 deficient mice exhibited little pathology in skeletal muscle $[5,6]$ while others reported a mild muscular dystrophy phenotype in a different strain of Ano5-KO mice, which also exhibited defective myoblast fusion and delayed muscle

\footnotetext{
*Correspondence: renzhi.han@osumc.edu

${ }^{1}$ Division of Cardiac Surgery, Department of Surgery, Davis Heart

and Lung Research Institute, The Ohio State University Wexner Medical Center, Columbus, OH 43210, USA

Full list of author information is available at the end of the article
}

regeneration after cardiotoxin injection [7]. In line with a potential role of ANO5 in muscle regeneration, the LGMDR12 patients manifested clinical symptoms that persisted longer than normal after a significant muscle injury [8]. Moreover, several recent studies revealed that Ano5 may participate in plasma membrane repair (PMR) through coordination with other membrane repair proteins such as dysferlin, annexin A1-A6, or regulating sarcoplasmic reticulum $\mathrm{Ca}^{2+}$ [7, 9-11]. Thus, defective PMR may also contribute to the development of muscular dystrophy caused by ANO5 mutations.

Defining specific ANO5 interactions and spatially or temporally restricted local proteomes would potentially improve our understanding of ANO5-regulated cellular processes. Recent technological advancements in proximity labeling with mutant biotin ligase variants such as BioID, BioID2, TurboID enable discovery of protein neighborhoods defining functional protein complexes 
and/or organelle compositions. Here we employed BioID2 to depict the Ano5 interactome and identified a novel Ano5-interacting protein, BVES (Blood Vessel Epicardial Substrance, also known as POPDC1), which is highly expressed in striated muscles. Interestingly, genetic mutations in BVES are linked to LGMDR25 and cardiac arrhythmia [12-17], suggesting that LGMDR12 and LGMDR25 may share some common pathogenic mechanisms.

\section{Results}

BioID2 proximity labeling of ANO5-interacting proteins in $\mathrm{C} 2 \mathrm{C} 12$ muscle cells

We employed proximity labeling with Bid-ID2 to identify ANO5-interacting proteins in $\mathrm{C} 2 \mathrm{C} 12$ muscle cells as this approach has the advantage to capture weak/ transient interactions with high sensitivity and low background contamination [18]. To increase experimental stringency, we used ANO6-BioID2 (an Ano5 paralogue) and BioID2-MG53 (a known PMR protein) as controls. C2C12 cells stably expressing ANO5-BioID2, ANO6BioID2 or BioID2-MG53 with FLAG or myc tag (Fig. 1A) were generated by lentiviral transduction. Western blot showed that all three transgenes were expressed well in their corresponding $\mathrm{C} 2 \mathrm{C} 12$ stable cell lines (Fig. 1B, C). To examine whether the BioID2 in the fusion proteins can biotinylate endogenous proteins, the stable cell lines were analyzed by Western blot using streptavidinHRP after incubation with or without $50 \mu \mathrm{M}$ biotin for $24 \mathrm{~h}$. As expected, the biotinylated proteins were readily
(A)

\begin{tabular}{|c|c|c|c|}
\hline FLAG & \multicolumn{2}{|c|}{ hANO5 } & BiolD2 \\
\hline & nAno6 & myc & BiolD2 \\
\hline myc & BiolD2 & $\mathrm{m}$ & G53 \\
\hline
\end{tabular}

(C)

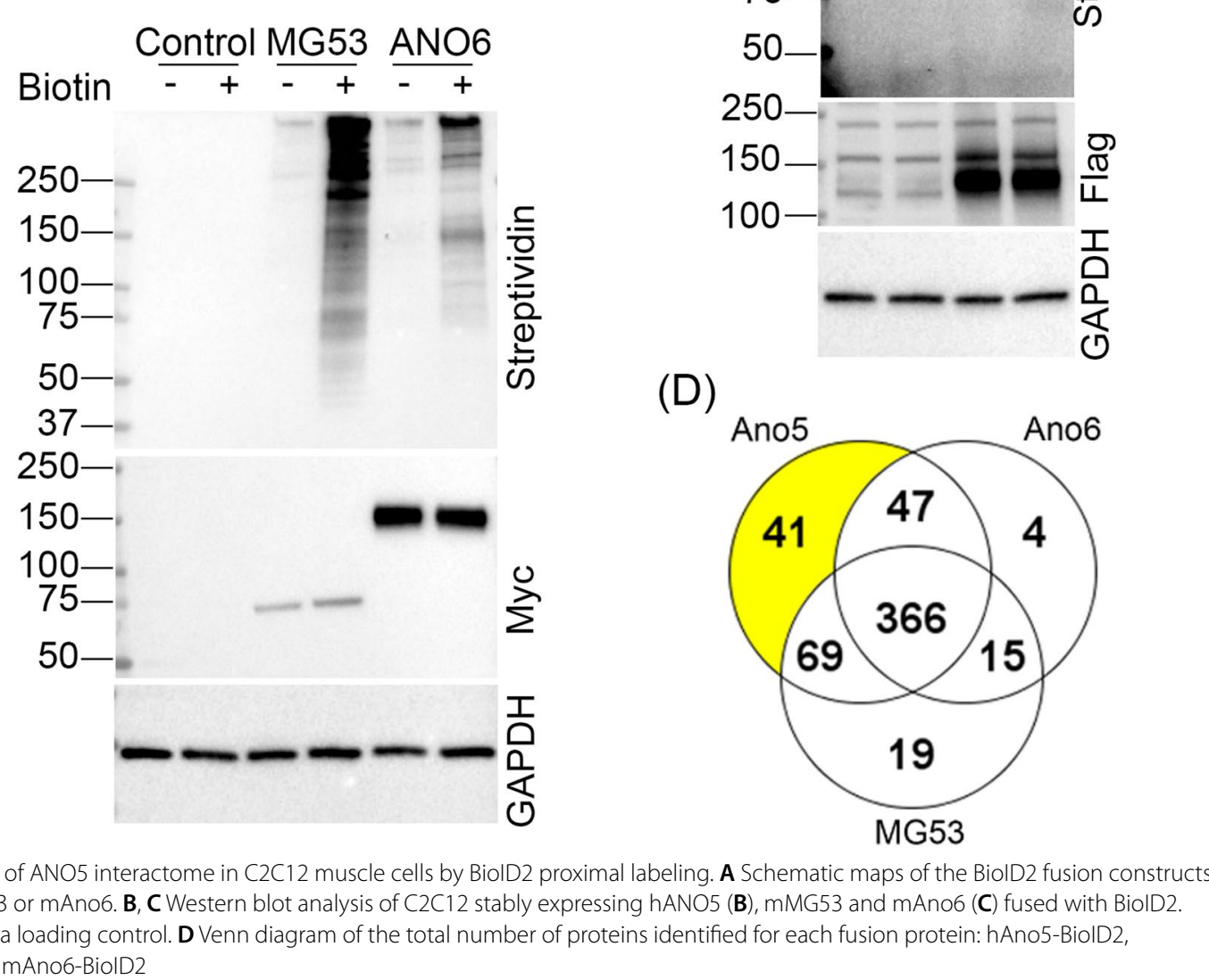

Fig. 1 Identification of ANO5 interactome in C2C12 muscle cells by BiolD2 proximal labeling. A Schematic maps of the BiolD2 fusion constructs with hANO5, mMG53 or mAno6. B, C Western blot analysis of C2C12 stably expressing hANO5 (B), mMG53 and mAno6 (C) fused with BiolD2. GAPDH was used as a loading control. D Venn diagram of the total number of proteins identified for each fusion protein: hAno5-BiolD2, BiolD2-mMG53, and mAno6-BiolD2
(B)

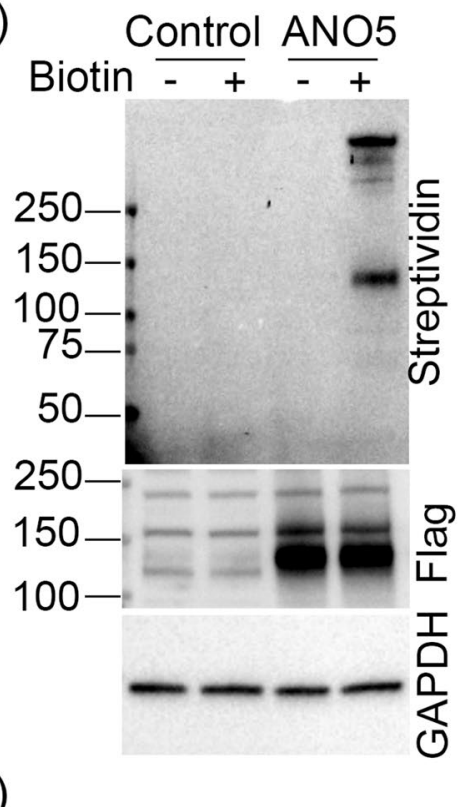


detectable in the lysates from the stable ANO5-BioID2, ANO6-BioID2 and BioID2-MG53 C2C12 cells with biotin incubation as compared to the cells without biotin treatment, which showed minimal background biotinylation similar to the control C2C12 sample (Fig. 1B, C).

The biotinylated proteins were isolated using streptavidin magnetic beads and subjected to mass spectrometry for protein identification. A total of 561 unique proteins with a minimum of 2 unique peptides and a false discovery rate (FDR) below $1.0 \%$ were identified from the three samples with 366 shared among them (Fig. 1D). Forty-one unique proteins were specifically identified in the ANO5-BioID2 sample (Fig. 1D, Additional file 1: Table S1).

\section{Validation of BVES-ANO5 interaction}

Among the unique proteins identified in the ANO5BioID2 sample, two members of the three POPDC family proteins including BVES and POPDC3 were of particular interest for the following reasons. Both genes were highly expressed in striated muscles [19-21] and their genetic mutations were linked to LGMD patients with or without cardiac involvement [22]. To validate the interaction between ANO5 and BVES, we performed Co-IP in COS-1 cells co-expressing ANO5 and myc-tagged BVES, POPDC2 or POPDC3. Preliminary experiment found that POPDC3 was poorly expressed in COS-1 cells (Additional file 1: Fig. S1) and thus it was not included in the final Co-IP experiment. As shown in Fig. 2A, ANO5 was specifically pulled down with BVES and POPDC2 by the anti-myc antibody. Similarly, this interaction also occurred in $\mathrm{C} 2 \mathrm{C} 12$ myoblasts stably expressing ANO5BioID2 transiently transfected with BVES-myc (Fig. 2B). We generated a series of truncations or deletions to map the interaction domains between ANO5 and BVES (Fig. 2C). Both full-length and the $\mathrm{N}$ terminal fragment of ANO5 were readily pulled down by BVES-myc (Fig. 2D). We further narrowed down the first 121 amino acids of ANO5 mediating the ANO5-BVES interaction (Fig. 2E). Similarly, the Co-IP studies with BVES deletion or truncation mutants showed that the $\mathrm{C}$-terminal region of BVES was involved in mediating the ANO5-BVES interaction. These biochemical data confirmed that BVES is a novel ANO5-interacting protein.

\section{Co-localization of ANO5 and BVES in muscle and non-muscle cells}

We performed immunofluorescence staining and confocal imaging to analyze the cellular localization of ANO5 and BVES. Substantial co-localization of ANO5 and BVES were observed in COS-1 cells (Fig. 3A), C2C12 myoblasts (Fig. 3B), C2C12 myotubes (Fig. 3C) and human myoblasts (Fig. 3D) co-expressing these two proteins. Consistent with previous studies showing that ANO5 was enriched at the ER membrane [23, 24], we observed that there was substantial fluorescence overlapping between GFP-ANO5 and ER tracker (Fig. 3E). Immunofluorescence staining also showed that BVESmyc was co-localized with the ER marker calnexin (Fig. 3F). In addition, we performed immunofluorescence staining of BVES and other subcellular markers. BVES was also found to be co-localized with the early endosome marker EEA1, but not with the markers for late endosome (Rab7), recycling endosome (Rab11) or lysosome (LAMP1) (Additional file 1: Fig. S2).

\section{Generation of Ano5- and Bves-KO C2C12 cells by genome editing}

Transcription activator like effector nuclease (TALEN)mediated genome editing was used to generate Ano5KO C2C12 cells. Two TALEN target sites were chosen from the exon 5 of mouse Ano5 gene and two pairs of TALENs (Fig. 4A) were assembled using the Goldengate TALEN kit as described before [25]. The TALEN plasmids were transfected into $\mathrm{C} 2 \mathrm{C} 12$ cells and genome editing was verified by T7E1 assay (Fig. 4B). As the E5ATALEN pair appeared to have a higher editing efficiency than the E5B-TALEN pair, we used the E5A-TALEN pair transfected $\mathrm{C} 2 \mathrm{C} 12$ cells for single cell colony derivation. Forty-eight hours after transfection with the E5A-TALEN pair, the $\mathrm{C} 2 \mathrm{C} 12$ cells were serially diluted for single cell clone screening. Individual clones were randomly picked up and screened by PCR and T7E1 assay. The clone 8 showed additional lower band besides the two cleavage bands of expected sizes from T7E1 digestion (Fig. 4C), and this additional band was found to carry a large 542bp deletion around the target site (Additional file 1: Fig. S3). The Clone 8 was chosen for further purification by serial dilutions. Twelve clones were then screened for the deletion PCR product and differentiation ability. The E5A8-12 clone was found to carry homozygous 542-bp deletion, which is expected to disrupt the reading frame of Ano5. This cell clone (designated as the Ano5-KO) can be differentiated to form myotubes (Additional file 1: Fig. S4). RT-PCR analysis confirmed that the Ano5 transcript expression was disrupted in the Ano5-KO C2C12 cells following differentiation (Fig. 4D).

We similarly established Bves-KO $\mathrm{C} 2 \mathrm{C} 12$ by using the state-of-the-art cytosine base editing (CBE) [26]. A premature stop codon was engineered into the coding exon 4 of Bves by transfection of $\mathrm{C} 2 \mathrm{C} 12$ cells with $\mathrm{CBE}$ and a targeting gRNA (Fig. 4E). After cell sorting, a single cell clone was confirmed to carry the homozygous Q159X mutation by Sanger sequencing (Fig. 4E). This cell clone (designated as Bves-KO) can be differentiated to form myotubes (Additional file 1: Fig. S5). 


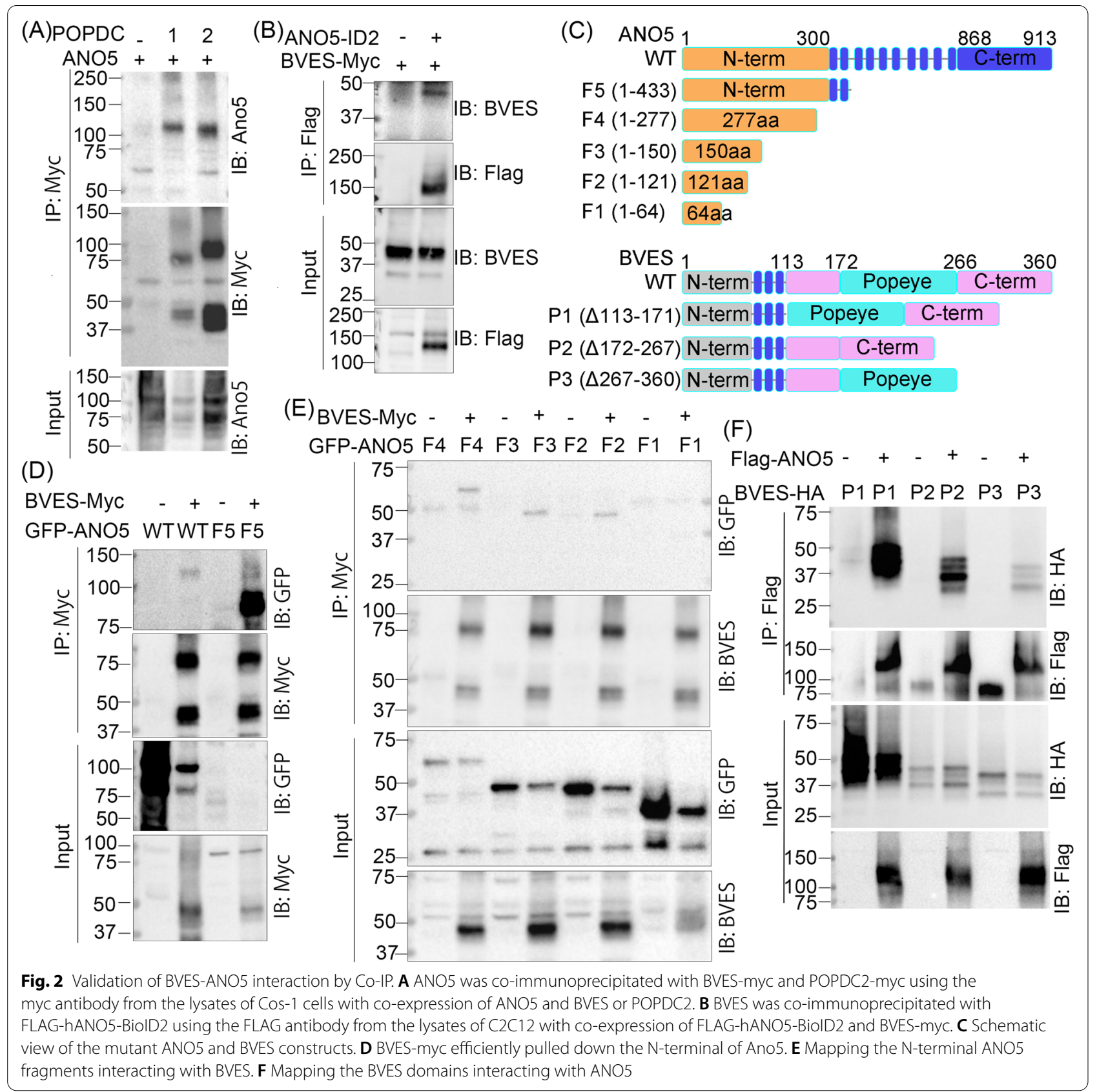

The loss of BVES expression was confirmed in BvesKO C2C12 myotubes by Western blot (Fig. 4F). Multiple BVES specific bands at around $41 \mathrm{kDa}$ and $60 \mathrm{kDa}$ appeared in WT myotubes, corresponding to the monomer and glycosylated form, and these bands were disrupted in Bves-KO myotubes. Disruption of Ano5 did not seem to alter the expression of BVES (Fig. 4F).
Impact of ANO5 or BVES deficiency on $\mathrm{C} 2 \mathrm{C} 12$ myoblast proliferation and differentiation

The expression of Ano5 and Bves during C2C12 myoblast differentiation was examined by quantitative RTPCR. As shown in Fig. 5A and B, the expression of both Ano5 and Bves were significantly increased during myoblast differentiation, suggesting that they may regulate 


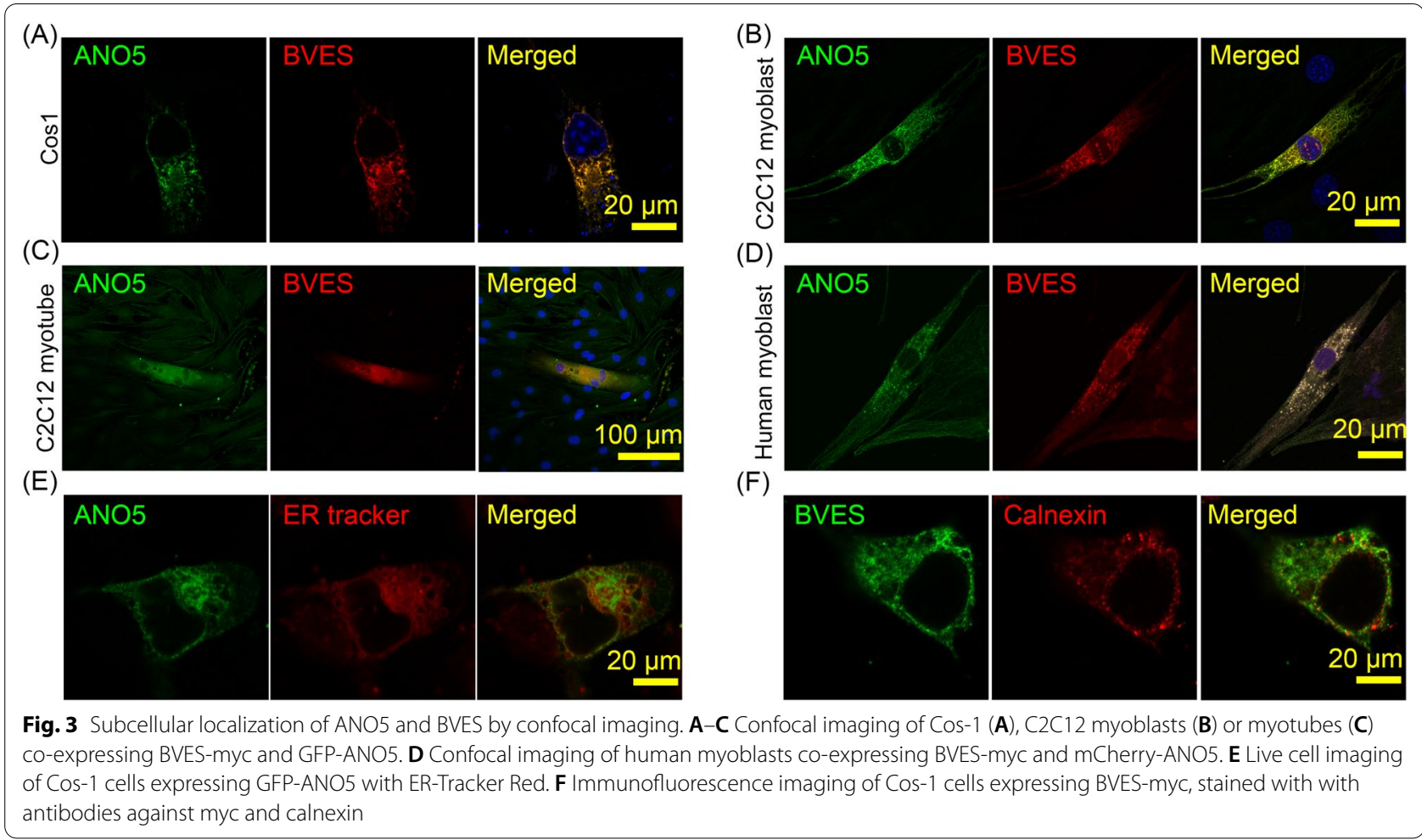

myogenesis. The CCK8 proliferation assay showed that Ano 5 or Bves gene disruption did not significantly affect myoblast proliferation (Fig. 5C). To examine the impact of Ano5 or Bves gene disruption on myoblast differentiation, the WT, Ano5-KO and Bves-KO C2C12 cells were differentiated for 4 days and stained with myosin heavy chain (MyHC) and DAPI (Fig. 5D). The fusion index (the percentage of myonuclei in $\mathrm{MyHC}+$ cells with $\geq 2$ nuclei/ cell) was significantly decreased in Ano5-KO and Bves$\mathrm{KO}$ cells as compared to the control cells (Fig. 5E), indicating that both ANO5 and BVES are indispensable for muscle differentiation.

\section{Discussion}

In this study, we identified BVES as a new ANO5-interacting protein in skeletal muscle cells using the BioID2 proximity labeling approach and mapped the interacting domains between BVES and ANO5. We further showed that genetic disruption of Bves or Ano5 with gene editing compromised $\mathrm{C} 2 \mathrm{C} 12$ myoblast differentiation.

BVES is one of the three POPDC proteins encoded in mammalian genome. All POPDC genes are highly expressed in striated muscles [20]. While BVES expressed at similar levels in both skeletal muscle and heart, $P O P D C 3$ is more selectively expressed in skeletal muscle and $P O P D C 2$ is more restricted to heart [27]. Consistent with their tissue expression profiles, patients that carry mutations in BVES develop LGMDR25 and AV-block of varying degree [12-17], whereas mutations in POPDC3 cause LGMD without affecting the heart [28] and the opposite is true for POPDC2 [29]. All three POPDC proteins are believed to act as cAMP effectors because they all carry a high-affinity cAMP binding Popeye domain [30]. Previous studies showed that the POPDC proteins regulate the mechano-gated potassium channel TREK-1 in a cAMP-dependent manner [28, 30,31]. However, it is unknown whether the alterations of the TREK-1 channel activity is directly responsible for muscular dystrophy and cardiac arrhythmia in the patients.

The physical interaction between ANO5 and BVES revealed in our study suggests that these proteins may participate in common cellular processes. Indeed, we showed that genetic disruption of Ano5 or Bves impaired myoblast differentiation in $\mathrm{C} 2 \mathrm{C} 12$ cells. In addition to regulating muscle differentiation, several recent studies demonstrated a role of ANO5 in PMR by regulating the SR calcium dynamics and coordinating with other PMR proteins [7, 9-11]. This raises an interesting possibility that BVES may also regulate PMR. If so, the cAMP signaling would very likely also be involved in the regulation of PMR. In supporting of this notion, BVES mutant LGMD patient muscle fibers showed the presence of plasma membrane discontinuities and submembraneous vacuoles as revealed by transmission electron microscopy 


\section{$(A)_{\text {E5A:TCCAGAGAAGTGAGCAcagcaaagactcggtcTTCTTCAGAGATGGAA }}$ TAL1 RVDs: HD HD NI NN NI NN NI NI NN NG NN NI NN HD NI TAL2 RVDs: NG HD HD NI NG HD NG HD NG NN NI NI NN NI NI}

\section{E5B:TCGACTTTGTCCTGTCCTacgtggaggatttgaagaAAGATGGAGAGCTGAAGGCA} TAL1 RVDs: HD NN NI HD NG NG NG NN NG HD HD NG NN NG HD HD NG TAL2 RVDs: NN HD HD NG NG HD NI NN HD NG HD NG HD HD NI NG HD NG NG

(B)

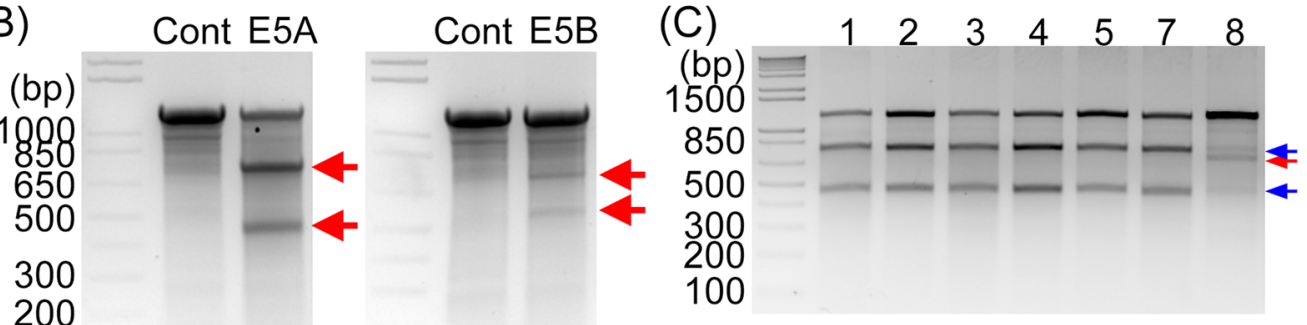

100

(E)

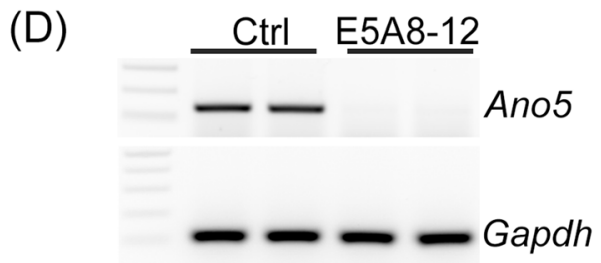

E) GGC CAG GTT TAC GCC ACA

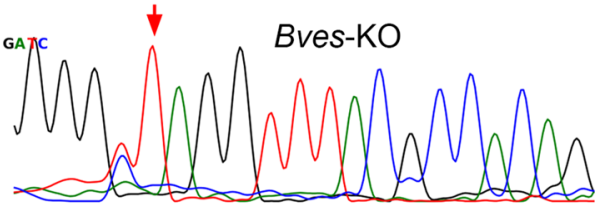

$(\mathrm{F})$
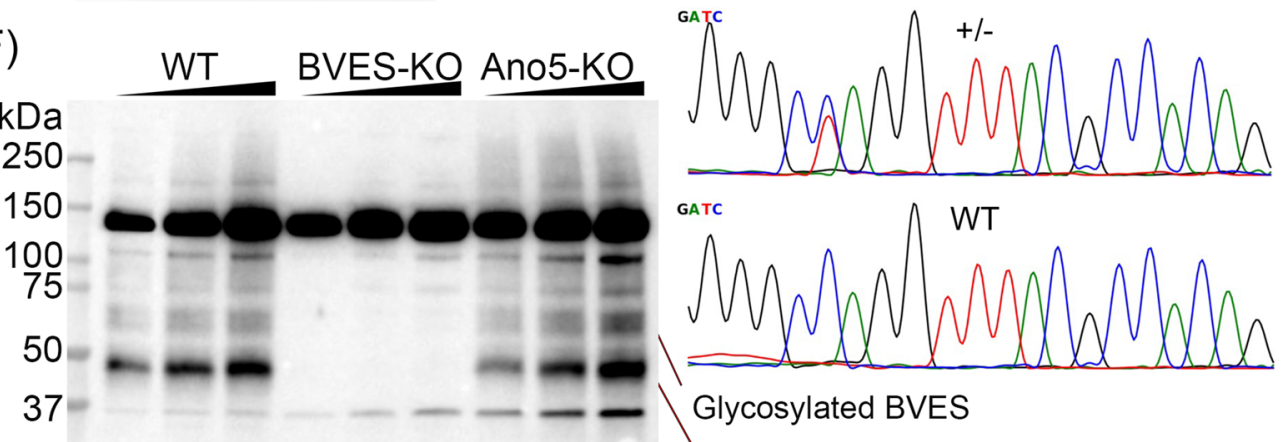

GATC

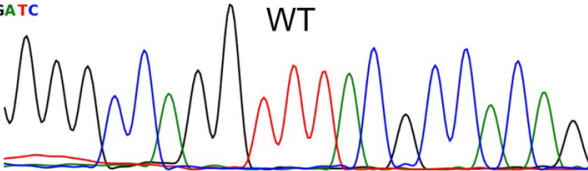

Glycosylated BVES

BVES

Fig. 4 Generation of Ano5-KO and BVES-KO C2C12 cells by gene editing. A Design of two TALEN pairs (E5A and E5B) targeting the exon 5 of mouse Ano5. The left and the right target sites were colored in red, with the repeat-variable di-residues (RVD) of each TAL shown below. B T7E1 assay of the gene editing activity conferred by E5A and E5B TALEN pairs in C2C12 myoblasts. The red arrows indicate the T7E1 enzyme cleavage bands shown in TALEN-transfected cells but not in control (Ctrl) samples. C Screening of individual C2C12 clones transfected with E5A TALEN pair by T7E1 assay. The blue arrows indicate the predicted T7E1 cleavage products. The red arrow indicates a smaller product appeared in Clone 8. D RT-PCR analysis of Ano5 expression in control and Clone E5A8-12. E Generation of Bves-KO C2C12 cells by CBE base editing. The gRNA targeting the exon 4 of mouse Bves was designed to install a premature TAG stop codon at position 159 (highlighted in red). Sanger sequencing confirmed the genotype of BVES-KO C2C12 cells. F Western blot analysis of BVES expression in the total membrane fractions of WT, BVES-KO and Ano5-KO C2C12 myoblasts

analysis [31]. As an important second messenger, cAMP has been implicated in many aspects of muscle physiology, such as glycogenolysis, contractility, sarcoplasmic calcium dynamics, and hypertrophy [32]. Interestingly, the cAMP-PKA signaling was found to activate ANO9's cation channel activity [33]. It is thus plausible to propose that BVES, as a cAMP effector protein, is involved in regulating ANO5's channel and lipid scrambling activities
$[34,35]$, thus facilitating proper execution of PMR, muscle differentiation and other unknown physiological processes.

In $\mathrm{C} 2 \mathrm{C} 12$ cells, BVES was found to be mainly localized to the internal membrane structures such as ER and early endosomes, whereas previous studies showed clear plasma membrane localization in isolated adult cardiac myocytes and human skeletal muscle [31,36]. It is not 


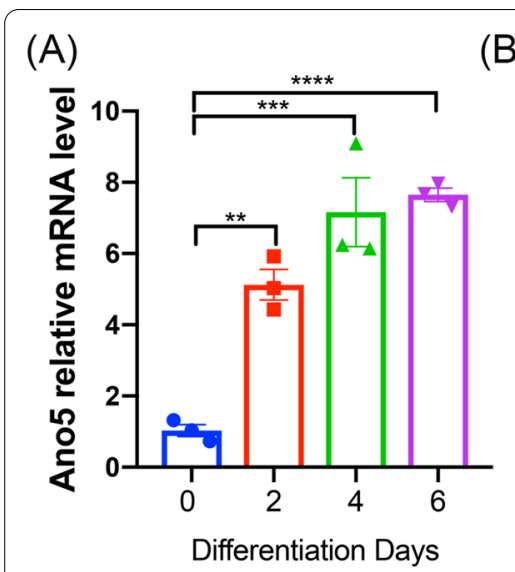

(D)

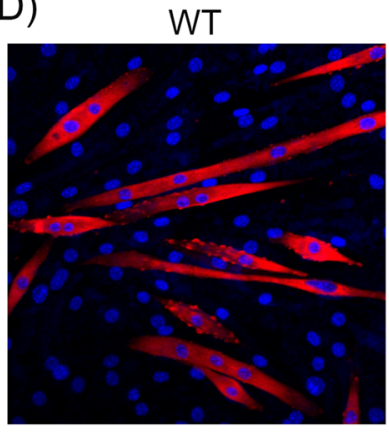

(B)

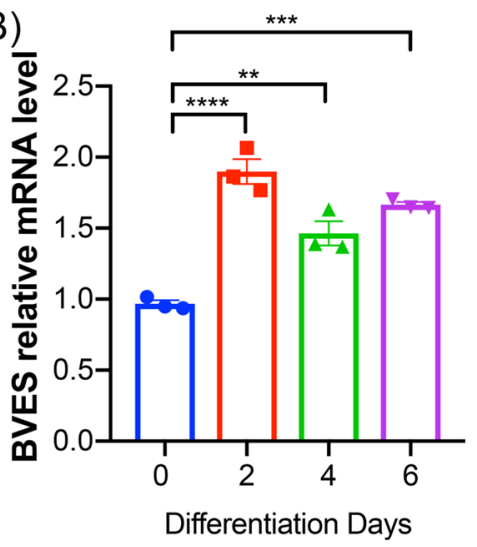

(C)

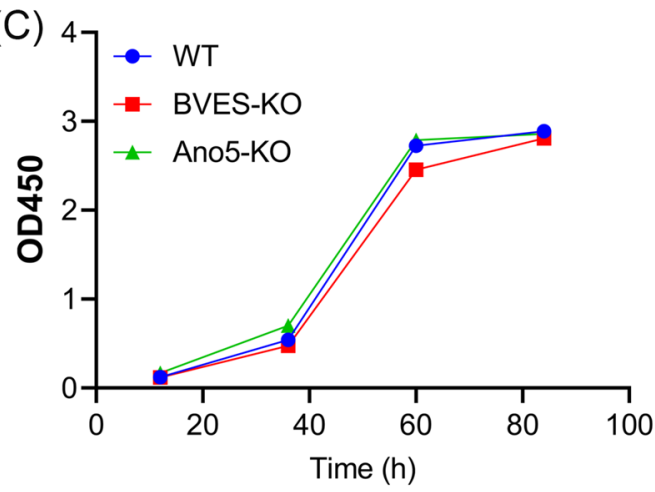

(E)
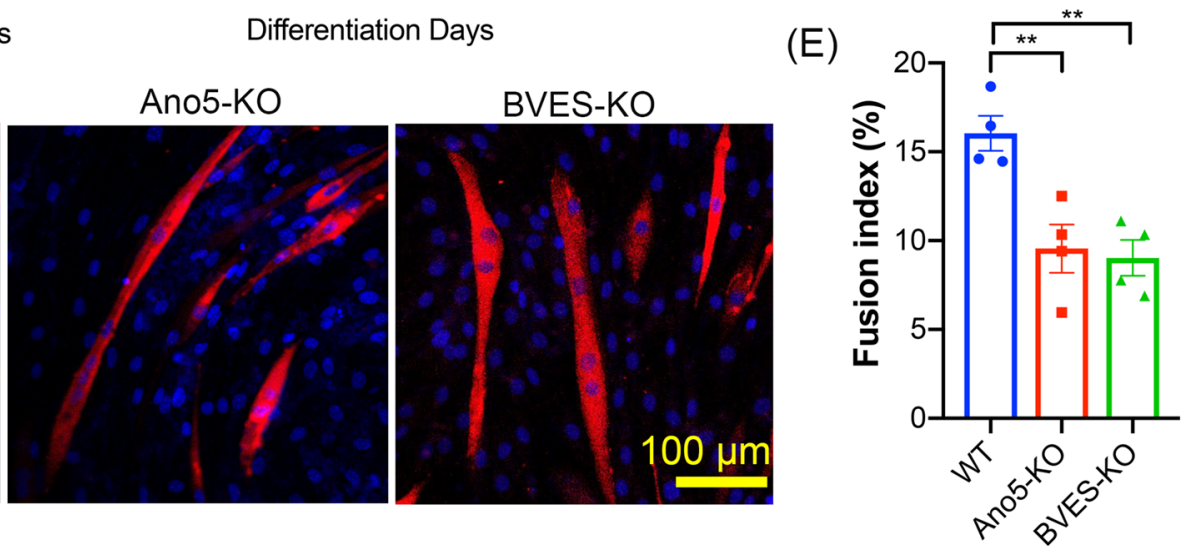

Fig. 5 Impact of BVES or ANO5 deficiency on myoblast proliferation and differentiation. A, B Quantitative RT-PCR analysis of Ano5 (A) and BVES (B) expression during C2C12 myoblast differentiation. ${ }^{* *} p<0.01,{ }^{* * *} p<0.001,{ }^{* * * *} p<0.0001$ (one-way ANOVA with Turkey's post tests). C The CCK8 proliferation assay of WT, BVES-KO and Ano5-KO C2C12 myoblasts from 12 to $84 \mathrm{~h}$ in culture. D Immunofluorescence images of WT, BVES-KO and Ano5-KO C2C12 myotubes on day 4 of differentiation stained with the monoclonal antibody against myosin heavy chain (MHC) and DAPI. Scale bar: $100 \mu \mathrm{m}$. E Quantification of the fusion index of WT, BVES-KO and Ano5-KO C2C12 myotubes in differentiation medium for four days. ${ }^{* *} \mathrm{p}<0.01$ (one-way ANOVA with Turkey's post tests)

clear what mechanism regulates the different cellular localization of BVES in C2C12 muscle cells versus adult muscle fibers. One possibility is the glycosylation status of BVES as protein glycosylation plays an important role in plasma membrane localization of transmembrane proteins. We found that BVES ran into $50-70 \mathrm{KDa}$ on SDSPAGE in addition to the $42 \mathrm{kDa}$ core peptide, consistent with previous reports that the Asn20 and Asn27 of BVES were glycosylated [37, 38]. Understanding the role of BVES glycosylation in muscle biology warrants future investigation.

\section{Conclusion}

In conclusion, we showed that BVES is a novel ANO5interacting protein, potentially participating in regulating common processes such as muscle differentiation and PMR. Future studies are required to dissect the molecular mechanisms for ANO5-BVES interaction in muscle biology and disease.

\section{Materials and methods}

\section{Plasmid construction}

The BioID2 was amplified by PCR from the Myc-BioID2MCS plasmid (Addgene \#74,223, Watertown, MA), fused with human $A N O 5$, mouse Ano6 or mouse $M g 53$ cDNA, and inserted into pLVX-puro (Clontech, San Jose, CA) to obtain pLVX-hANO5-BioID2, pLVX-mAno6-BioID2, and pLVX-BioID2-mMG53, respectively. BVES-myc, POPDC2-myc and POPDC3 were constructed by inserting the corresponding cDNA into pCDNA3.1-myc backbone. ANO5 related plasmids were described previously [39]. The truncation or deletion mutant BVES constructs were generated by overlapping PCR. The Ano5-targeting TALEN pairs were assembled using the Golden Gate TALEN kit (Addgene \#1000000016) as previously described [25] and the TALEN pairs were coupled with 2A peptide [40]. The protein sequences of Ano5TALENs are provided in the Additional file 1: Fig. S6. pCMV-AncBE4max plasmid was obtained from Addgene (\#112094). The pCMV-AncBE4-GFP was generated by 
ligation of SacI-EcoR1 fragment from pCMV-AncBE4 max and the EcoRI/AgeI-digested GFP fragment into SacI/AgeI linearized pCMV-AncBE4max backbone. The annealed gRNA oligos (targeting mouse Bves) were cloned into pLenti-OgRNA-Zeo plasmid as previously described [41, 42]. All plasmids used in this study are listed in Additional file 1: Table S2.

\section{Cell culture and transfection}

HEK293 and COS-1 cells were cultured in Dulbecco's modified Eagle medium (DMEM, GIBCO) containing $1 \mathrm{~g} / \mathrm{l}$ glucose and supplemented with $10 \%(\mathrm{v} / \mathrm{v})$ fetal bovine serum (FBS, Sigma) and $1 \%(\mathrm{v} / \mathrm{v})$ penicillin-streptomycin (Pen-Strep, Sigma-Aldrich). C2C12 and human myoblasts were grown in DMEM with $20 \%$ FBS and differentiated in DMEM with $2 \%$ horse serum after $70-80 \%$ confluency. All cells were cultured in a $37{ }^{\circ} \mathrm{C}$ incubator with a humidified $5 \% \mathrm{CO}_{2}$ atmosphere. Transfection of HEK293 or COS-1 cells were performed using $\mathrm{X}$-tremeGENE ${ }^{\mathrm{TM}} \mathrm{HP}$ DNA transfection reagent (\#6366244001, Sigma-Aldrich, St. Louis, MO). For $\mathrm{C} 2 \mathrm{C} 12$ and human myoblasts, plasmids were transfected with the Neon electroporation system (Thermo Scientific, NY). For confocal imaging, the aforementioned cells were plated onto collagen-coated $35-\mathrm{mm}$ glass-bottom dishes post transfection and grown for 24-48 $\mathrm{h}$.

\section{Generation of Bves-KO and Ano5-KO C2C12 cells}

To generate Ano5-KO C2C12 cells, the $\mathrm{C} 2 \mathrm{C} 12$ cells were electroporated with pTAL9-Ano5E5A and individual cell clones were screened by PCR and T7E1 assay. To generate Bves-KO $\mathrm{C} 2 \mathrm{C} 12$ cell line, the $\mathrm{C} 2 \mathrm{C} 12$ cells were sorted for GFP into single cells in a 96-well plate after electroporation with the pCMV-AncBE4-GFP and pLenti-BvesgRNA-Zeo. The expanded individual cell clones were screened by PCR and Sanger sequencing.

\section{BiolD2 pull-down}

For BioID2 pull down, C2C12 myotubes stably expressing hANO5-BioID2, mAno6-BioID2 or BioID2-mMG53 were incubated with $50 \mu \mathrm{M}$ biotin for $16 \mathrm{~h}$. After washing the cells with PBS twice very gently, the cells were lysed in $2.4 \mathrm{ml}$ lysis buffer (50 mM Tris, $\mathrm{pH} 7.4,500 \mathrm{mM}$ $\mathrm{NaCl}, 0.4 \%$ SDS, $1 \mathrm{mM}$ dithiothreitol, and $1 \times$ complete protease inhibitor). Triton X-100 was added to $2 \%$ final concentration. After sonication, an equal volume of $50 \mathrm{mM}$ Tris ( $\mathrm{pH}$ 7.4) was added. After centrifugation at $16,500 \times \mathrm{g}$ for $10 \mathrm{~min}$, the supernatant was collected to a $15-\mathrm{mL}$ tube and incubated with $300 \mathrm{ul}$ magnetic streptavidin beads (\#88,816, Thermo Scientific, NY) overnight at $4{ }^{\circ} \mathrm{C}$. Beads were washed according to the following steps: twice with $2 \%$ SDS, once with wash buffer containing $0.1 \%$ deoxycholate, $1 \%$ Triton X-100, $500 \mathrm{mM} \mathrm{NaCl}$,
$1 \mathrm{mM}$ EDTA, and $50 \mathrm{mM}$ HEPES, $\mathrm{pH} 7.5$, once with wash buffer containing $250 \mathrm{mM} \mathrm{LiCl}, 0.5 \% \mathrm{NP}-40,0.5 \%$ deoxycholate, $1 \mathrm{mM}$ EDTA, and $10 \mathrm{mM}$ Tris, $\mathrm{pH}$ 8, and once with $50 \mathrm{mM}$ Tris $\mathrm{pH}$ 8. Ten percent of the samples were saved for Western blot analysis. The other $90 \%$ of the samples were subjected to mass spectrometry analysis at the Ohio State University Comprehensive Cancer Center Proteomic Shared Resources.

\section{Cell proliferation assay}

The cell proliferation was examined by the CCK- 8 cell counting kit (Dojindo Molecular Technologies, MD, USA). WT, BVES-KO or Ano5-KO C2C12 cells were prepared in 96-well plates at the initial density of $5 \times 10^{3}$ cells/well. The $450 \mathrm{~nm}$ absorbance was measured at $12-84 \mathrm{~h}$ according to the manufacturer's instructions.

\section{Western blot}

The cells were lysed with cold RIPA buffer supplemented with protease inhibitors, and the extracted proteins were quantified by $\mathrm{DC}^{\mathrm{TM}}$ Protein Assay Reagent (Bio-Rad Laboratories, Hercules, CA). The extracted protein samples were separated by stain-free SDS-PAGE gels (Bio-Rad Laboratories, 4-15\%) and transferred onto Nitrocellulose Membranes $(0.45 \mu \mathrm{m})$. Primary antibodies include the rabbit polyclonal anti-BVES (1:1000, HPA014788, Sigma-Aldrich, St. Louis, MO), mouse monoclonal antiAno5 (1:1000, N421A/85, UC Davis/NIH NeuroMab Facility, Davis, CA), anti-GAPDH (1:4000, MAB374, Cell Signaling Technology, Danvers, MA), anti-GFP antibody $(1: 1000$, A01388, Genscript, Piscataway, NJ), streptavidin-HRP (1:20, DY998, R\&D Systems, Minneapolis, MN), myc (1:1000, \#2276, Cell Signaling Technology, Danvers, MA), FLAG (1:1000, \#F3165, Sigma-Aldrich, St. Louis, MO) and HA (1:1000, \#3724, Cell Signaling Technology, Danvers, MA). Secondary HRP-conjugated goat anti-mouse (1:4000), goat anti-rabbit (1:4000) antibodies were obtained from Cell Signaling Technology. The membranes were developed using ECL western blotting substrate (Pierce Biotechnology, Rockford, IL) and images were scanned with the ChemiDoc XRS + system (Bio-Rad Laboratories). Western blots were quantified using Image Lab 6.0.1 software (Bio-Rad Laboratories) according to the manufacturer's instructions.

\section{Immunofluorescence staining}

Cells were fixed with $4 \%$ paraformaldehyde for $10 \mathrm{~min}$ at room temperature. After washing with PBS, the slides were blocked with 5\% BSA with $0.3 \%$ Triton X-100 for $1 \mathrm{~h}$. The slides were incubated with primary antibodies at $4{ }^{\circ} \mathrm{C}$ overnight. The primary antibodies include myc (1:200, \#2276, Cell Signaling Technology, Danvers, MA), calnexin (1:200, \#2679, Cell Signaling Technology, 
Danvers, MA), and myosin heavy chain (1:200, MF20, Developmental Studies Hybridoma Bank, Iowa City, IA). The cells were then washed extensively with PBS and incubated with Alexa Fluor 568 (donkey anti-rabbit IgG, Invitrogen) or Alexa Fluor 488 (goat anti-rabbit IgG, Invitrogen) for $1 \mathrm{~h}$ at room temperature. The stained cells were sealed with VECTASHIELD Antifade Mounting Medium with DAPI (Vector Laboratory, Burlingame, CA). ER-tracker red (E34250, Thermo Scientific) was used for labeling endoplasmic reticulum in COS-1 cells after $24 \mathrm{~h}$ transfection with GFP-Ano5. All images were taken with a Zeiss 780 confocal microscope (Jena, Germany).

\section{Co-immunoprecipitation assay}

Cells were transfected with the indicated plasmids and lysed in RIPA lysis buffer [ $25 \mathrm{mM}$ Tris- $\mathrm{HCl}$ ( $\mathrm{pH} 7.4$ ), $150 \mathrm{mM} \mathrm{NaCl}, 5 \%$ Glycerol, 1\% Triton X-100, $2 \mathrm{mM}$ EDTA, and $1 \mathrm{mM}$ DTT supplemented with protease inhibitor cocktail (Roche)] at $48 \mathrm{~h}$ after transfection. Immunoprecipitation was performed by incubation with the indicated primary antibodies for $4 \mathrm{~h}$ and protein $\mathrm{A} / \mathrm{G}$ agarose beads (\#20423, Thermo Scientific) overnight at $4{ }^{\circ} \mathrm{C}$. The beads were washed at least three times with RIPA lysis buffer. Lysates and immunoprecipitated sampless were examined by using the indicated primary antibodies followed by the related secondary antibodies and the SuperSignal Chemiluminescence Kit (Thermo Fisher Scientific, Waltham, MA, USA).

\section{RNA extraction and quantitative RT-PCR analysis}

Total RNA was extracted from $\mathrm{C} 2 \mathrm{C} 12$ myoblasts or myotubes with Trizol. First-strand cDNA was synthesized using RevertAid RT Reverse Transcription Kit (Life Technologies, Carlsbad, CA, USA). Real-time PCR was performed using PerfeCTa SYBR Green FastMix (QuantaBio, USA) in CFX384 Real-time PCR Detection Systems (Bio-Rad). Samples were normalized for expression of GAPDH and analyzed by the $2^{-\Delta \Delta \mathrm{Ct}}$ method.

\section{Statistical analysis}

All in vitro experimental data were repeated a minimum of three times. Data are expressed as mean \pm the standard error of the mean (S.E.M.). Statistical differences were determined by two-tailed, unpaired Student's $t$ test for two groups or one-way ANOVA with Turkey's post tests for multiple group comparisons using Prism 8 (Graphpad Software, La Jolla, California). A $P$ value $<0.05$ was considered to be significant.
Co-immunoprecipitation; ER: Endoplasmic reticulum; FDR: False discovery rate; GDD: Gnathodiaphyseal dysplasia; gRNA: Guide RNA; KO: Knock out; LGMDR: Limb girdle muscular dystrophies recessive; MMD3: Miyoshi myopathy type 3; MyHC: Myosin heavy chain; PMR: Plasma membrane repair; POPDC: Popeye domain-containing protein; TALEN: Transcription activator like effector nuclease; WT: Wild type.

\section{Supplementary Information}

The online version contains supplementary material available at https://doi. org/10.1186/s13578-021-00735-w.

Additional file 1: Table S1. List of unique proteins identified by hANO5BiolD2. Table S2. List of plasmids used in this study. Figure S1. Western blot analysis of exogenous expression of human. BVES, POPDC2 and POPDC3 in COS-1 cells. Figure S2. Immunofluorescence staining images of COS-1 cells expressing BVES-myc and GFP markers for endosomes and lysosomes. Figure S3. Sequence alignment showing the 542bp deletion in the genomic DNA of the E5A8-12 Clone. Figure S4. Photographs of WT and Ano5-KO C2C12 cells differentiated for 5 days. Scale bar: 100 $\mu \mathrm{m}$. Figure S5. Differentiation of WT and Bves-KO C2C12 cells differentiated for 5 days. Scale bar: 200 Mm. Figure S6. Amino acid sequences of Ano5E5-TALEN and Ano5E5ATALEN.

\section{Acknowledgements}

The authors would like to thank all lab members for their constructive comments and suggestions.

\section{Authors' contributions}

$\mathrm{RH}$ conceived the study and wrote the manuscript. $\mathrm{HL}$ performed the experiments and participated in drafting the manuscript. $H L, Y G, Y Z$ and $L X$ constructed plasmids used in this study. SG assisted with confocal imaging. All authors have read and approved the final manuscript.

Funding

R.H. is supported by US National Institutes of Health grants (R01HL116546, R01HL159900, R01AR070752) and a Parent Project Muscular Dystrophy award.

Availability of data and materials

All relevant data supporting the key findings of this study are available within the article and its Additional file 1 or from the corresponding author upon reasonable request.

\section{Declarations}

Ethics approval and consent to participate Not applicable.

\section{Consent for publication}

All co-authors have reviewed and approved of the manuscript prior to submission. The manuscript has been submitted solely to this journal and is not published, in press, or submitted elsewhere.

\section{Competing interests}

The authors declare that they have no conflict of interests.

\section{Author details}

${ }^{1}$ Division of Cardiac Surgery, Department of Surgery, Davis Heart and Lung Research Institute, The Ohio State University Wexner Medical Center, Columbus, OH 43210, USA. ² Department of Pediatrics, The Third Xiangya Hospital, Central South University, Changsha, China.

Received: 28 October 2021 Accepted: 17 December 2021 Published online: 28 December 2021 


\section{References}

1. Oh U, Jung J. Cellular functions of TMEM16/anoctamin. Pflugers Arch. 2016;468(3):443-53.

2. Suzuki J, Fujii T, Imao T, Ishihara K, Kuba H, Nagata S. Calcium-dependent phospholipid scramblase activity of TMEM16 protein family members. J Biol Chem. 2013;288(19):13305-16.

3. Duran C, Hartzell HC. Physiological roles and diseases of Tmem16/ Anoctamin proteins: are they all chloride channels? Acta Pharmacol Sin. 2011:32(6):685-92.

4. Dhoke NR, Kim H, Selvaraj S, Azzag K, Zhou H, Oliveira NAJ, et al. A universal gene correction approach for FKRP-associated dystroglycanopathies to enable autologous cell therapy. Cell Rep. 2021;36(2): 109360.

5. Xu J, El Refaey M, Xu L, Zhao L, Gao Y, Floyd K, et al. Genetic disruption of Ano5 in mice does not recapitulate human ANO5-deficient muscular dystrophy. Skeletal Muscle. 2015;5:43.

6. Gyobu S, Miyata H, Ikawa M, Yamazaki D, Takeshima H, Suzuki J, et al. A role of TMEM16E carrying a scrambling domain in sperm motility. Mol Cell Biol. 2016:36(4):645-59.

7. Griffin DA, Johnson RW, Whitlock JM, Pozsgai ER, Heller KN, Grose WE, et al. Defective membrane fusion and repair in Anoctamin5-deficient muscular dystrophy. Hum Mol Genet. 2016;25(10):1900-11.

8. Milone M, Liewluck T, Winder TL, Pianosi PT. Amyloidosis and exercise intolerance in ANO5 muscular dystrophy. Neuromuscul Disord. 2012;22(1):13-5.

9. Foltz SJ, Cui YY, Choo HJ, Hartzell HC. ANO5 ensures trafficking of annexins in wounded myofibers. J Cell Biol. 2021. https://doi.org/10.1083/jcb. 202007059.

10. Chandra G, Sreetama SC, Mazala DAG, Charton K, VanderMeulen JH, Richard I, et al. Endoplasmic reticulum maintains ion homeostasis required for plasma membrane repair. J Cell Biol. 2021. https://doi.org/10.1083/jcb. 202006035

11. Chandra G, Defour A, Mamchoui K, Pandey K, Mishra S, Mouly V, et al. Dysregulated calcium homeostasis prevents plasma membrane repair in Anoctamin 5/TMEM16E-deficient patient muscle cells. Cell Death Discov. 2019:5:118.

12. De Palma C, Morisi F, Cheli S, Pambianco S, Cappello V, Vezzoli M, et al. Autophagy as a new therapeutic target in Duchenne muscular dystrophy. Cell Death Dis. 2012;3: e418.

13. Indrawati LA, lida A, Tanaka Y, Honma Y, Mizoguchi K, Yamaguchi T, et al. Two Japanese LGMDR25 patients with a biallelic recurrent nonsense variant of BVES. Neuromuscul Disord. 2020;30(8):674-9.

14. De Ridder W, Nelson I, Asselbergh B, De Paepe B, Beuvin M, Ben Yaou R, et al. Muscular dystrophy with arrhythmia caused by loss-of-function mutations in BVES. Neurol Genet. 2019;5(2): e321.

15. Chu ML, Moran E. The limb-girdle muscular dystrophies: is treatment on the horizon? Neurotherapeutics. 2018;15(4):849-62.

16. Taghizadeh E, Rezaee M, Barreto GE, Sahebkar A. Prevalence, pathological mechanisms, and genetic basis of limb-girdle muscular dystrophies: a review. J Cell Physiol. 2019;234(6):7874-84.

17. Becerra SP, Koczot F, Fabisch P, Rose JA. Synthesis of adeno-associated virus structural proteins requires both alternative mRNA splicing and alternative initiations from a single transcript. J Virol. 1988;62(8):2745-54.

18. Trinkle-Mulcahy L. Recent advances in proximity-based labeling methods for interactome mapping. F1000Res. 2019. https://doi.org/10.12688/ f1000research.16903.1.

19. Reese DE, Zavaljevski M, Streiff NL, Bader D. bves: a novel gene expressed during coronary blood vessel development. Dev Biol. 1999;209(1):159-71.

20. Andree B, Hillemann T, Kessler-Icekson G, Schmitt-John T, Jockusch $\mathrm{H}$, Arnold $\mathrm{HH}$, et al. Isolation and characterization of the novel popeye gene family expressed in skeletal muscle and heart. Dev Biol. 2000;223(2):371-82

21. Osler ME, Smith TK, Bader DM. Bves, a member of the Popeye domaincontaining gene family. Dev Dyn. 2006;235(3):586-93.

22. Amunjela JN, Swan AH, Brand T. The role of the Popeye domain containing gene family in organ homeostasis. Cells. 2019;8(12):1594. https://doi. org/10.3390/cells8121594.

23. Beecher G, Tang C, Liewluck T. Severe adolescent-onset limb-girdle muscular dystrophy due to a novel homozygous nonsense BVES variant. J Neurol Sci. 2021;420: 117259

24. Whitlock JM, Yu K, Cui YY, Hartzell HC. Anoctamin 5/TMEM16E facilitates muscle precursor cell fusion. J Gen Physiol. 2018;150(11):1498-509.
25. Xu L, Zhao P, Mariano A, Han R. Targeted myostatin gene editing in multiple mammalian species directed by a single pair of TALE nucleases. Mol Ther Nucleic Acids. 2013;2: e112.

26. Komor AC, Kim YB, Packer MS, Zuris JA, Liu DR. Programmable editing of a target base in genomic DNA without double-stranded DNA cleavage. Nature. 2016;533(7603):420.

27. Brand T. The Popeye domain containing genes and their function as CAMP effector proteins in striated muscle. J Cardiovasc Dev Dis. 2018:5(1):18. https://doi.org/10.3390/jcdd5010018.

28. Vissing J, Johnson K, Topf A, Nafissi S, Diaz-Manera J, French VM, et al. POPDC3 gene variants associate with a new form of limb girdle muscular dystrophy. Ann Neurol. 2019;86(6):832-43.

29. Rinne S, Ortiz-Bonnin B, Stallmeyer B, Schindler RFR, Kiper AK, Dittmann $\mathrm{S}$, et al. Conduction disorder caused by a mutation in POPDC2, a novel modulator of the cardiac sodium channel SCN5A. Acta Physiol. 2016;216.

30. Froese A, Breher SS, Waldeyer C, Schindler RF, Nikolaev VO, Rinne S, et al. Popeye domain containing proteins are essential for stressmediated modulation of cardiac pacemaking in mice. J Clin Invest. 2012;122(3):1119-30.

31. Schindler RF, Scotton C, Zhang J, Passarelli C, Ortiz-Bonnin B, Simrick S, et al. POPDC1(S201F) causes muscular dystrophy and arrhythmia by affecting protein trafficking. J Clin Invest. 2016;126(1):239-53.

32. Berdeaux R, Stewart R. CAMP signaling in skeletal muscle adaptation: hypertrophy, metabolism, and regeneration. Am J Physiol Endocrinol Metab. 2012;303(1):E1-17.

33. Kim H, Kim H, Lee J, Lee B, Kim HR, Jung J, et al. Anoctamin 9/TMEM16J is a cation channel activated by CAMP/PKA signal. Cell Calcium. 2018;71:75-85

34. Whitlock JM, Hartzell HC. Anoctamins/TMEM16 proteins: chloride channels flirting with lipids and extracellular vesicles. Annu Rev Physiol. 2017;79:119-43.

35. Falzone ME, Malvezzi M, Lee BC, Accardi A. Known structures and unknown mechanisms of TMEM16 scramblases and channels. J Gen Physiol. 2018;150(7):933-47.

36. Alcalay $Y$, Hochhauser E, Kliminski V, Dick J, Zahalka MA, Parnes D, et al Popeye domain containing 1 (Popdc1/Bves) is a caveolae-associated protein involved in ischemia tolerance. PLOS ONE. 2013;8(9): e71100.

37. Schindler RF, Scotton C, French V, Ferlini A, Brand T. The Popeye domain containing genes and their function in striated muscle. J Cardiovasc Dev Dis. 2016;3(2):22. https://doi.org/10.3390/jcdd3020022.

38. Knight RF, Bader DM, Backstrom JR. Membrane topology of Bves/Pop1A, a cell adhesion molecule that displays dynamic changes in cellular distribution during development. J Biol Chem. 2003;278(35):32872-9.

39. Xu J, Xu L, Lau YS, Gao Y, Moore SA, Han R. A novel ANO5 splicing variant in a LGMD2L patient leads to production of a truncated aggregationprone Ano5 peptide. J Pathol Clin Res. 2018;4(2):135-45.

40. Mariano A, Xu L, Han R. Highly efficient genome editing via 2A-coupled co-expression of two TALEN monomers. BMC Res Notes. 2014;7:628.

41. Wang P, Xu L, Gao Y, Han R. BEON: a functional fluorescence reporter for quantification and enrichment of adenine base-editing activity. Mol Ther 2020;28(7):1696-705

42. Xu L, Zhang C, Li H, Wang P, Gao Y, Mokadam NA, et al. Efficient precise in vivo base editing in adult dystrophic mice. Nat Commun. 2021;12(1):3719.

\section{Publisher's Note}

Springer Nature remains neutral with regard to jurisdictional claims in published maps and institutional affiliations. 